\title{
ACTIVATION CALCULATIONS FOR THE TARGET OF A SPALLATION ULTRA-COLD NEUTRON SOURCE AT PSI
}

\author{
Michael Wohlmuther* and Jan Züllig \\ Paul-Scherrer Institut, CH-5232 Villigen PSI, Switzerland
}

\begin{abstract}
A spallation ultra-cold neutron source-UCN source-is scheduled to start operation at PSI in 2006 using up to 2 mA $590 \mathrm{MeV}$ protons from the ring cyclotron. It will be operated in a pulsed mode with an average current of $20 \mu \mathrm{A}$. For safe maintenance, during operation as well as handling, transport and storage of the UCN target assembly after its lifespan, detailed knowledge about the activation induced by the impinging protons and secondary radiation fields is required. The Monte Carlo transport code MCNPX was coupled with the European Activation System-EASY-to calculate the residual nuclide production in the UCN target assembly. The nuclide inventory is finally used to design the shielded exchange flask that is needed to safely remove and transport the UCN target assembly after its lifespan to a hotcell for dismantling.
\end{abstract}

\section{INTRODUCTION}

A new type of ultra-cold neutron (UCN) source based on the spallation process is scheduled to start operation at PSI in $2006^{(1-4)}$. The essential elements of the UCN source are the spallation target assembly, a large heavy-water moderator of about $\sim 4 \mathrm{~m}^{3}$ at room temperature, a converter system of 30 litre of solid deuterium $\left(\mathrm{SD}_{2}\right)$ at a low temperature $(\sim 6$ $\mathrm{K})$ for the production of ultra-cold neutrons ${ }^{(5)}$ and a storage volume for the ultra-cold neutrons (see Figure 1). The source will be operated in a pulsed mode, with a $590 \mathrm{MeV}$ proton beam of up to $2 \mathrm{~mA}$ on for $8 \mathrm{~s}$ and off for $792 \mathrm{~s}$, resulting in an average current of $20 \mu \mathrm{A}$. Pulsed operation brings two advantages: (1) reduced average power deposition in the $\mathrm{SD}_{2}$ so it can be located in a high-flux region and (2) reduced background at the experiments as the measurements will be made during the beam-off phase. The target assembly will be mounted horizontally in the proton beam. It consists of the Zircaloy target, a lead block and an aluminium container with integrated beam window (target head), as well as a steel shield towards the handling zone (see Figure 1). Since the components of the target head lie directly under the $\mathrm{SD}_{2}$ moderator, they have to be made from materials with low absorption cross sections to minimise neutron flux depression. The target is slightly longer than the range of $590 \mathrm{MeV}$ protons and $\sim 70-80 \%$ of the beam power will be deposited in the Zircaloy. Owing to the large amounts of energy deposited, especially in the Zircaloy rod target, the target head will be cooled with $\mathrm{D}_{2} \mathrm{O}$. The lead block behind the Zircaloy structure is the first part of the shielding in the forward direction. The target shaft $(\sim 2 \mathrm{~m})$ is made of steel, to reduce the induced activation in the handling region so that hands-on maintenance is possible after an adequate cooling time.

*Corresponding author: michael.wohlmuther@psi.ch
Spallation neutrons - mainly produced in the Zircaloy-will be thermalised in the $\mathrm{D}_{2} \mathrm{O}$ premoderator, further cooled in the $\mathrm{SD}_{2}$ and finally, some of them downscattered so that they enter the storage volume in the ultra-cold neutron energy regime $\left(E_{\mathrm{kin}} \leq 250 \mathrm{neV}\right)$. During one proton pulse, equilibrium is reached between produced and reabsorbed ultra-cold neutrons in the storage volume above the $\mathrm{SD}_{2}$. Monte Carlo calculations show that an average ultra-cold neutron density of $\sim 10^{3} \mathrm{~cm}^{-3}$ can be delivered to the experiments ${ }^{(2)}$ with this source. To ensure safe maintenance and operation of this facility, detailed knowledge about power density distributions and activation of all components of the system is required. Concerning activation, special attention has to be paid to the target assembly. It is the most highly activated component of the system because of direct exposure to the primary proton beam.

In case of maintenance or exchange (it is planned to replace the Zircaloy target by a lead Cannelloni target after a few years of operation), the target assembly has to be transported to the hotcell of PSI-West for the necessary work. The shielded exchange flask for the transport will be designed on the basis of the calculated induced activation of the target assembly. In this report, an overview of the method for the activation calculation will be given, followed by the results for the activation of the UCN source target assembly. Finally, basic parameters for the design of the shielded exchange flask will be discussed.

\section{CALCULATION METHOD}

Activation calculations have two distinct parts: (1) the estimate of nuclide production rates and (2) solving the Bateman equations for the system.

The target assembly of the UCN source will be activated by a mixed radiation field consisting of the primary $590 \mathrm{MeV}$ protons, secondary high-energy 


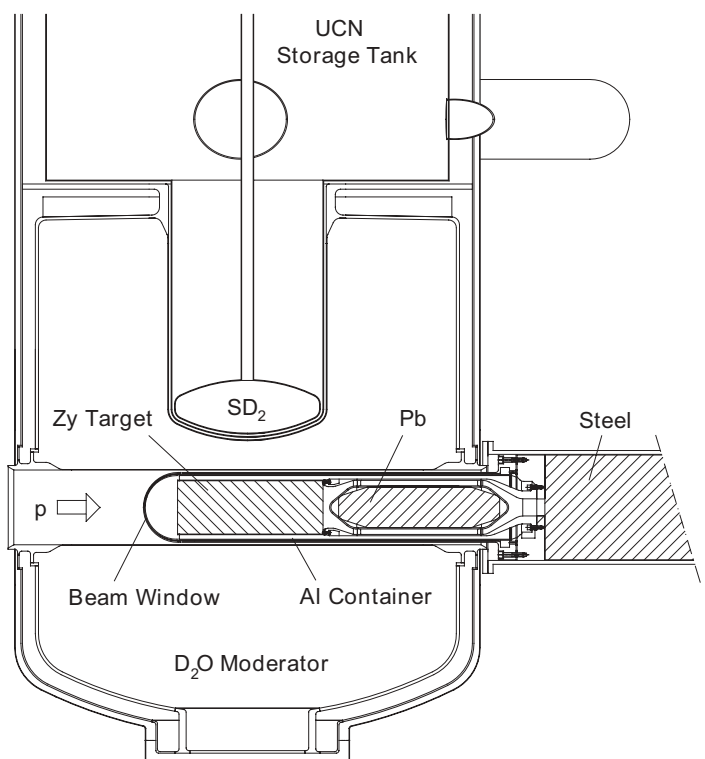

Figure 1. Schematic drawing of the UCN target assembly.

collision products $(E>20 \mathrm{MeV})$ and low-energy neutrons $(E<20 \mathrm{MeV})$. To account for these different 'sources' of activation the calculation is split into two parts. In the first step, the Monte Carlo particle transport code MCNPX ${ }^{(6)}$ is used to determine the nuclide inventory produced by the primary protons as well as by the high-energy $(E>20 \mathrm{MeV})$ secondary particles created in spallation reactions within the target assembly. Moreover, low-energy neutron spectra $(E<20 \mathrm{MeV})$ are calculated for each component of the target assembly. These neutron spectra can then be used in conjunction with the European activation system-EASY ${ }^{(7)}$. This code is designed for fusion applications and able to calculate the activation induced by neutron fluxes with energies $<20 \mathrm{MeV}$. EASY has two parts: (1) the inventory code FISPACT ${ }^{(8)}$ solving the Bateman equations of the system and (2) several neutron cross section and decay libraries, the so-called EAF libraries ${ }^{(9)}$. FISPACT uses the EAF libraries to calculate the amount of isotopes either at a specific time during irradiation with neutrons $<20 \mathrm{MeV}$ or after a decay time following shutdown. The nuclide production rates calculated with MCNPX in the physics model region $(E>20 \mathrm{MeV})$ are now combined with the nuclide inventory produced by low-energy neutrons $(E<20 \mathrm{MeV})$ calculated with FISPACT. This extension of FISPACT has been implemented by Petrovich $^{(10)}$ and was called SP-FISPACT. To account for the interactions of particles with energies $>20 \mathrm{MeV}$ - calculated with MCNPX - 'pseudo cross sections' are introduced in SP-FISPACT. The

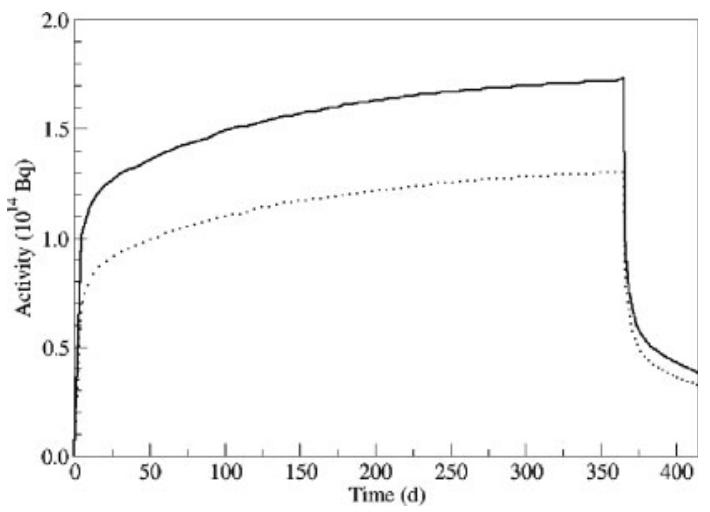

Figure 2. Total induced activation (solid line) and activation due to high-energy $(E>20 \mathrm{MeV})$ particle interactions (dotted line) in the Zircaloy target block as a function of time. An average proton current of $20 \mu \mathrm{A}$ is assumed to hit the target for $1 \mathrm{y}$. Thereafter, the behaviour of activity over $50 \mathrm{~d}$ cooling is shown.

activation due to low-energy neutrons is calculated by folding the neutron fluxes obtained with MCNPX and the appropriate activation cross sections of the EAF libraries. Thus, using the combination of MCNPX and SP-FISPACT, nuclide production rates can be calculated spanning the whole energy regime of interest.

\section{RESULTS}

Calculations, as described in the previous section, have been performed for each of the components of the UCN target assembly. However, in this report only an overview of the results can be given. A listing of the nuclide inventory for each component of the UCN target would be too extensive. Therefore, only total activities and leading isotopes will be discussed for the major parts of the target assembly.

The component with the highest induced activation is the Zircaloy target, since the primary proton beam is fully stopped within this module. The primary protons induce spallation reactions which form an internuclear cascade consisting of high-energy nucleons, clusters and pions as well as an evaporation spectrum. The high-energy particles undergo further spallation reactions within the various regions of the target assembly, while the low-energy neutrons will produce residual nuclei through reaction channels like $(n, \alpha),(n, \gamma),(n, 2 n)$, etc. with masses close to the nuclei hit.

In Figure 2, one can see the total activity (solid line) and the activity induced by high-energy particles $(E>20 \mathrm{MeV})$ (dotted line) in the Zircaloy part of the target assembly as a function of time. It is assumed that the target is irradiated with an average current of $20 \mu \mathrm{A}$ for $1 \mathrm{y}$, after which the beam is 
switched off and the decay of the activity is observed. The maximum activity is $1.7 \times 10^{14} \mathrm{~Bq}$. One can see that the total activity of the Zircaloy rods is dominated by that from high-energy particles; $75 \%$ is due to residuals produced in high-energy reactions. Moreover, the lifetimes of the isotopes produced in low-energy neutron reactions are short, since after $50 \mathrm{~d}$ cooling the total activity has dropped nearly to the level of the activity induced by high-energy particles. The steep decline of activity within the first days after shut-down is mainly due to the decay of metastable $\left({ }^{90} \mathrm{Zr}^{\mathrm{m}},{ }^{89} \mathrm{Y}^{\mathrm{m}}\right)$ and short-lived yttrium and zirconium isotopes. After $30 \mathrm{~d}$ decay, the activity induced in the Zircaloy has dropped by a factor of 3 . The nuclides dominating the activity in the Zircaloy rods after a cooling time of $30 \mathrm{~d}$ are ${ }^{88} \mathrm{Y}$, ${ }^{88} \mathrm{Zr}$ and ${ }^{85} \mathrm{Sr}$, which together contribute $>50 \%$ to the total activity. These isotopes are produced in high-energy reactions in the Zircaloy rods. The main isotope predominantly produced by lowenergy neutron activation is ${ }^{95} \mathrm{Nb}$ with an activity of $3.810^{12} \mathrm{~Bq}$.

The values for the total activation of the Zircaloy have been compared to results for the SINQ Zircaloy target ${ }^{(11)}$ calculated with HETC + O5R_PSI ${ }^{(12)}$ in combination with ORIHET ${ }^{(13)}$. Agreement within a factor of 2 was found.

In Table 1, the total activities for the different components of the UCN target assembly are given for different cooling times. It should be noted that the values for the steel shield were calculated for the first $55 \mathrm{~cm}$ of steel only; for parts towards the end of the steel shield a decrease of activity is observed.

For the other components of the target assembly, the following leading isotopes can be identified after a cooling time of $30 \mathrm{~d}$. For the beam window and the target container which are made of aluminium, the isotope with the highest contribution to the activity is ${ }^{22} \mathrm{Na}$. Since this isotope is produced in high-energy reactions, the contribution of ${ }^{22} \mathrm{Na}$ is higher in the beam window $(80 \%)$ than in the container $(45 \%)$. For the $\mathrm{Pb}$ block which is located downstream from the Zircaloy rods, ${ }^{124} \mathrm{Sb}$ and ${ }^{195} \mathrm{Au}$ give the highest contributions with 29.8 and $19.6 \%$ of the total activity, respectively. The leading nuclides in

Table 1. Total activities $(\mathrm{Bq})$ after irradiation for $1 \mathrm{y}$ with an average proton current of $20 \mu \mathrm{A}$ within different regions of the UCN target assembly for cooling times of 1, 10 and $30 \mathrm{~d}$.

\begin{tabular}{lccc}
\hline Component & $1 \mathrm{~d}$ & $10 \mathrm{~d}$ & $30 \mathrm{~d}$ \\
\hline Zircaloy & $1.05 \times 10^{14}$ & $5.83 \times 10^{13}$ & $4.47 \times 10^{13}$ \\
Al container & $1.73 \times 10^{11}$ & $5.67 \times 10^{10}$ & $5.35 \times 10^{10}$ \\
Al beam window & $7.37 \times 10^{10}$ & $3.90 \times 10^{10}$ & $3.62 \times 10^{10}$ \\
Pb block & $6.03 \times 10^{11}$ & $1.59 \times 10^{11}$ & $9.26 \times 10^{10}$ \\
Steel $(55 \mathrm{~cm})$ & $4.25 \times 10^{11}$ & $3.61 \times 10^{11}$ & $3.14 \times 10^{11}$ \\
\hline
\end{tabular}

the first $55 \mathrm{~cm}$ of the steel shield are ${ }^{55} \mathrm{Fe}(41.0 \%)$ and ${ }^{54} \mathrm{Mn}(37.8 \%)$.

Several of the isotopes produced during the irradiation will decay by emitting gammas of specific energies. The resulting gamma spectra for the aluminium beam window are shown in Figure 3, for cooling times of 1 and $30 \mathrm{~d}$. After $30 \mathrm{~d}$, the gamma line in the interval between 2.5 and $3.0 \mathrm{MeV}$, due to ${ }^{24} \mathrm{Na}$, has disappeared because the half-life of the isotope is $14.96 \mathrm{~h}$.

The isotopic composition calculated for each component of the UCN target assembly was finally used to design the shield of the UCN target transport flask. The main criterion for the exchange flask is that the dose rate must not exceed $2 \mathrm{~m} \mathrm{~Sv} \mathrm{~h}^{-1}$ on the outer surface and $0.1 \mathrm{~m} \mathrm{~Sv} \mathrm{~h}^{-1}$ at a distance of 1 $\mathrm{m}^{(14)}$. The calculations were performed with the program MICROSHIELD ${ }^{(15)}$. Since only one shieldsource combination can be calculated with MICROSHIELD at the same time, dose rates were calculated separately for each component of the target assembly. The shielding material was chosen to be steel. In the region of the Zircaloy rods, a thickness of $30 \mathrm{~cm}$ will be needed. After a length of $135 \mathrm{~cm}$ the thickness is reduced to $25 \mathrm{~cm}$. Where the steel of the target shaft begins, the shielding of the exchange flask will again be decreased to $8 \mathrm{~cm}$, and after an additional $55 \mathrm{~cm}$ to $5 \mathrm{~cm}$ thickness. The maximum dose rate was calculated to be $0.68 \mathrm{mSv} \mathrm{h}^{-1}$ at the surface and $0.1 \mathrm{~m} \mathrm{~Sv} \mathrm{~h}^{-1}$ in $1 \mathrm{~m}$ distance.

\section{SUMMARY}

Nuclide inventories have been calculated for the different components of the UCN target assembly using a combination of the Monte Carlo transport

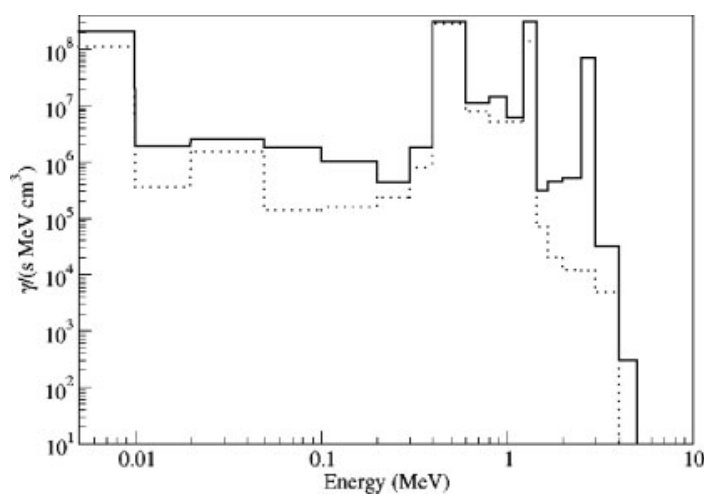

Figure 3. Calculated decay gamma spectra for the aluminium beam window after irradiation for $1 \mathrm{y}$ with 20 $\mu \mathrm{A}$ proton beam and cooling of $1 \mathrm{~d}$ (solid line) and $30 \mathrm{~d}$ (dotted line). 


\section{ACTIVATION CALCULATIONS FOR AN UCN SOURCE}

code MCNPX and the European activation system (EASY).

With the knowledge of the isotopic composition for the different parts of the UCN target assembly after an irradiation of $1 \mathrm{y}$ with an average proton current of $20 \mu \mathrm{A}$, the basic parameters for the shield of the UCN target exchange flask were calculated using the MICROSHIELD program. These parameters can now be used for the detailed design of the transport flask.

\section{REFERENCES}

1. Serebrov, A. P., Mityukhlyaev, V. A., Zakharov, A. A., Bowles, T., Greene, G. and Sromicki, J. Solid deuterium source of ultracold neutrons based on a spallation source. JETP Lett. 66(12), 802 (1997).

2. Fomin, A. et al. An ultracold neutron facility at PSI. Internal PSI Report TM-14-01-01. Paul Scherrer Institut, CH-5232 Villigen PSI, Switzerland, (2000).

3. Atchison, F. et al. An ultracold neutron facility at PSI. PSI Scientific Report 2002, Vol. 1, ISSN 1423-7296 (2003). Paul Scherrer Institut, CH-5232 Villigen PSI, Switzerland.

4. Kirch, K. for the UCN collaboration. An ultracold neutron facility at PSI. AIP Conference proceedings 610, International Nuclear Physics Conference INPC 2001, Berkley, CA, p. 997 (2001).

5. Serebrov, A. et al. Studies of a solid-deuterium source for ultra-cold neutrons. Nucl. Instrum. Meth. A 440, 685 (2000).

6. Waters, L. S. et al. MCNPX User's Manual Version 2.4.0. LA-CP-02-408. Los Alamos National
Laboratory, Los Alamos, NM 87545, USA, (2002). Available at http://mcnpx.lanl.gov

7. Forrest, R. A. EASY-99 Overview. EASY Documentation Series EDS-0, Culham Science Centre (1998).

8. Forrest, R. A. and Sublet, J.-Ch. FISPACT-99: User manual. EASY Documentation Series, UKAEA FUS 407, Culham Science Centre (1998).

9. Forrest, R. A. and Kopecky, J. The European Activation File: EAF-2001 cross section library. EASY Documentation Series, UKAEA FUS 451, Culham Science Centre (2001) In: The European Activation File: EAF-2001 decay library. Forrest, R. A. Ed., EASY Documentation Series, UKAEA FUS 452, Culham Science Centre (2001).

10. Petrovich, C. SP-FISPACT, a computer code for activation and decay calculations for intermediate energies. A connection of FISPACT and MCNPX. RT/ERG/ 2001/10 (ENEA: Bologna) (2001). Paul Scherrer Institut, CH-5232 Villigen PSI, Switzerland, PSI Internal Report.

11. Atchison, F. Activation of the SINQ Zircaloy rod target. PSI Internal Report, SINQ Project Report, SINQ/816/ AF30-302.- Paul Scherrer Institut, CH-5232 Villigen PSI, Switzerland (1993).

12. Atchison, F. and Pepin, M. The HETC Package for the $V A X$ (1984).

13. Atchison, F. and Schaal, H. Orihet 3-Version 1.12, A guide for users, private communication, (2001).

14. Bundesamt für Strassen Europäisches Übereinkommen über die Beförderung gefährlicher Güter auf der Strasse (ADR), Vol. 1, Ch. 2.2.7 (2001).

15. Microshield, Version 6, User's Manual, Grove Engineering, Rockville, MD (2003). 